Derecho \& Realidad

Núm. 23 • I semestre de 2014

Facultad de Derecho y Ciencias Sociales, UPTC

ISSN: 1692-3936

\title{
Mecanismos de protección de los Derechos Humanos en Colombia desde la pedagogía
}

\author{
Mechanisms of human rights protection in \\ Colombia from pedagogy
}

\author{
José Eriberto Cifuentes Medina** \\ Jairo Alberto Higuera Sandoval ${ }^{* * *}$
}

\section{Resumen}

Se pretende compartir una experiencia pedagógica sobre el manejo de los mecanismos de protección de los derechos humanos en Colombia, desarrollada en las asignaturas de Cultura Política y Ética con los estudiantes de Regencia de Farmacia, a fin de fortalecer conocimientos respecto a la Constitución Política de Colombia, instituciones y formas jurídicas de protección.

Esta propuesta de reflexión pedagógica se desarrolló en tres fases: la primera se dio en torno a la fundamentación teórica de los derechos humanos y mecanismos de protección, desde las diferentes perspectivas; en la segunda se diseñaron algunas estrategias de

* El texto es producto de la ampliación de una ponencia aceptada y presentada en el "XV Congreso Internacional de Filosofía Latinoamericana, Memoria, justicia y utopía. Diálogos filosóficos e interdisciplinarios", celebrado del 2 a 5 de julio de 2013 en la Universidad Santo Tomás, Bogotá, Colombia.

** Magíster (C) en Educación, Universidad Santo Tomás. Especialista en Educación con énfasis en Evaluación Educativa, Universidad Santo Tomás. Licenciado (C) en Teología, Universidad Santo Tomás. Licenciado en Filosofía y Educación Religiosa, Universidad Santo Tomás. Docente Escuela de Ciencias Humanísticas y de Educación, Facultad de Estudios a Distancia Uptc. Docente investigador del grupo GICl. Correo electrónico: eribertocifuentes@hotmail.com

*** Magíster en Administración de empresas, Universidad Externado de Colombia. Especialista en Finanzas, Universidad Pedagógica y Tecnológica de Colombia. Especialista en Sistemas, Universidad Nacional de Colombia. Especialista en Gestión Pública, Escuela Superior de Administración Pública ESAP. Economista de la Universidad Pedagógica y Tecnológica de Colombia. Docente ocasional de tiempo completo de la Facultad de Estudios a Distancia FESAD, área administrativa de la Uptc, sede Tunja. Correo electrónico: Jairo_higuera@yahoo.com 
aprehensión de los siguientes mecanismos: derecho de petición, acción de tutela, habeas corpus, habeas data, acción popular y acción de grupo y de las instituciones: Personería, Defensoría del pueblo y Procuraduría, de acuerdo con la Constitución de 1991; y la tercera fase correspondió al desarrollo de actividades de casos hipotéticos y reflexión pedagógica de la temática. Con miras a fortalecer la educación ciudadana de los estudiantes, resaltar el valor de la justicia y la igualdad, para que el ejercicio de los mecanismos para proteger, al menos los derechos fundamentales, no sea una utopía, más cuando se trata de personas que trabajan en el sector de la salud.

Los resultados han sido valiosos para el ejercicio de la labor docente respecto a la fundamentación de los derechos humanos, manejo de los mecanismos, al igual que el acercamiento a la Constitución de 1991. En cuanto al proceso formativo de los estudiantes, se vislumbraron logros en redacción, apropiación, manejo de las formas jurídicas. A partir de esta propuesta ellos son capaces de afrontar la defensa de los derechos humanos, la fundamentación teórica, ya que son jóvenes abiertos al conocimiento y a la reflexión, capaces de convertir las utopías en vías posibles de testimonio y ejercicio de la memoria.

\section{Palabras clave}

Mecanismos de protección, derechos humanos, Colombia.

\footnotetext{
Abstract

We aim to share an educational experience the mechanisms of protection of human rights in Colombia, developed in the subjects of Political Culture and Ethics with the students of Regency of Pharmacy, in order to strong knowledge about the Constitution of Colombia, institutions and legal forms of protection.

The proposed pedagogical reflection was implemented in three phases: the firs occurred on the theoretical foundation of human rights and «mechanisms of protection», from different perspectives; in the second one some apprehension of strategies of the following mechanisms were designed: right to petition, action of protection, habeas corpus, habeas data, popular action and group action and of the institutions: Office of the Solicitor, and Ombudsman Office, according to the 1991 Constitution, and the third phase corresponds to developing scenarios and activities of the thematic educational thinking, in order to strong the education of students, to highlight the value of justice and equality, so that the exercise of mechanisms to protect, at least the fundamental rights, is not utopian, especially when it comes to people working in the health sector.

The results have been valuable for teaching exercise on grounds of human rights instructional management mechanisms like 1991 Constitution. In terms of the learning process of students, it was envisioned achievements in writing, appropriation and management of legal forms. From this proposal they are able to deal with the defense of human rights, the theoretical found action, because they are open to knowledge and reflection, capable of making utopias in possible avenues of witness and memory exercise.

\section{Key words}

Mechanisms of protection, human rights, Colombia.
} 


\section{Introducción}

Se pretende compartir una experiencia sobre el manejo de los mecanismos de protección de los derechos humanos en Colombia, desarrollada con los estudiantes de Regencia de Farmacia, a fin de fortalecer conocimientos respecto a la Constitución Política, instituciones y formas jurídicas de protección.

El ejercicio investigativo parte del diseño y elaboración de una encuesta que busca evidenciar el dominio que sobre este tema tienen los estudiantes. Después se formula una pregunta a través del foro, en donde la participación de ellos permite avanzar en la profundización del tema. Finalmente se abordan los siguientes temas: a) breve fundamentación de cada mecanismo de protección de los derechos humanos y de las instituciones, b) diseño de estrategias para la aprehensión de cada mecanismo, como mapas conceptuales, entre otras y c) una guía para presentar cada mecanismo y casos hipotéticos.

En el desarrollo de este proceso investigativo con los estudiantes de educación superior, se nota el desconocimiento de los mecanismos de protección de los derechos humanos en Colombia.

\section{Objetivos:}

Objetivo general: identificar desde la pedagogía, el manejo de los mecanismos de protección de los derechos humanos y las estrategias para fomentar su dominio.

\section{Objetivos específicos:}

1. Diseñar un instrumento que permita observar el conocimiento que se tiene sobre los mecanismos de protección de los derechos humanos.

2. Plantear estrategias para el manejo y apropiación de los mecanismos de los derechos humanos en Colombia.

\section{Referentes teóricos}

Los derechos humanos son el conjunto de normas y recomendaciones para que cada persona de una sociedad promueva el respeto y una serie de valores por medio de la enseñanza, educación y práctica que van encaminados a la dignidad humana y a una excelente convivencia. Tomando como referencia estos fundamentos se encuentran los siguientes pasos. En primera instancia se halla «el ser», lo cual significa las respuestas a las preguntas de ¿Quién eres? ¿Qué quieres? ¿A dónde quieres llegar? En segundo lugar está «el ser humano», en este paso se puede encontrar ya de una manera más física al hombre. El tercer paso es la «dignidad», 
el valor espiritual que cada persona requiere para alcanzar la felicidad. Y el cuarto y último es «el deber ser del ser humano», las responsabilidades que son básicas para llegar a la felicidad cumpliendo deberes frente a la sociedad.

Se definen de manera breve los derechos humanos, que para su profundización se ahondará en el estudio de la Constitución a partir del artículo 11, en donde se encuentran «los derechos fundamentales, sociales, económicos, culturales, colectivos y del medio ambiente».

El Estado creó en la Constitución de 1991 un conjunto de instituciones y formas jurídicas para la protección de los derechos humanos:

Las personerías municipales: las personerías tienen la función de recibir e investigar inicialmente las denuncias sobre violaciones de derechos humanos.

La Defensoría del Pueblo: creada a partir de la Constitución de 1991, su función constitucional es velar por la promoción, el ejercicio y la divulgación de los derechos humanos. La Defensoría no cumple funciones investigativas, pero se ha encargado de recopilar denuncias que son pasadas posteriormente a la Procuraduría, que hace la investigación y define la sanción respectiva.

La Procuraduría: como la misión de la Procuraduría es la de vigilar la conducta de los empleados oficiales y el estricto cumplimiento de la ley y teniendo en cuenta que dentro de la Constitución se contempla la protección por parte del Estado de los derechos fundamentales de todos los ciudadanos colombianos, la procuraduría desempeña un papel importante cuando de abusos de poder se trata.

Existen las siguientes formas constitucionales de protección de los derechos humanos: 


\begin{tabular}{|c|l|}
\hline MECANISMO & \multicolumn{1}{|c|}{ DEFINICIÓN } \\
\hline $\begin{array}{c}\text { 1. Acción de } \\
\text { tutela Art. 86 }\end{array}$ & $\begin{array}{l}\text { Protege los Derechos fundamentales vulnerados o amenazadas por } \\
\text { acción u omisión por cualquier autoridad pública o particulares que } \\
\text { cumplan funciones públicas. }\end{array}$ \\
\hline $\begin{array}{c}\text { El Derecho } \\
\text { de petición }\end{array}$ & Sirve para solicitar información \\
\hline $\begin{array}{c}\text { El Habeas } \\
\text { Corpus (Art.30) }\end{array}$ & $\begin{array}{l}\text { Protege el derecho a la libertad. Derecho a que se resuelva en el } \\
\text { término de 36 horas la situación jurídica a quien estuviese privado } \\
\text { de la libertad. }\end{array}$ \\
\hline $\begin{array}{c}\text { El Habeas } \\
\text { Data }\end{array}$ & $\begin{array}{l}\text { Protege el derecho al buen nombre y sacar a la persona de bases de } \\
\text { datos de deudores morosos. Tienen derecho a conocer, actualizare y } \\
\text { rectificar las informaciones en bancos de datos, protege su derecho } \\
\text { a la intimidad personal y familiar y a su buen nombre. El estado } \\
\text { debe respetarlo hacerlo respetar. }\end{array}$ \\
\hline $\begin{array}{c}\text { Acción de } \\
\text { cumplimiento }\end{array}$ & $\begin{array}{l}\text { Sirve para hacer cumplir el fallo de una ley Hacer efectivo el } \\
\text { cumplimiento de una "ley o un acto administrativo", para, derechos } \\
\text { en peligro de ser vulnerados o amenazados por acción u omisión de } \\
\text { las autoridades. }\end{array}$ \\
\hline Acción popular & $\begin{array}{l}\text { Defiende a un grupo de personas. Protegen derechos e intereses } \\
\text { colectivos, relacionados con el patrimonio, el espacio, la seguridad } \\
\text { y la salubridad pública, la moral administrativa, el ambiente, la libre } \\
\text { competencia económica y similar reglados por la ley o los que originan } \\
\text { daño a un número plural de personas. }\end{array}$ \\
Busca indemnización por daños causados \\
\hline Acción de grupo
\end{tabular}

Estrategias didácticas y organizadores gráficos. "La comunicación humana, a través de gráficos, es parte de nuestra historia. Las pinturas rupestres y representaciones en las cuevas y en otros lugares muestran el uso de estos medios como forma de comunicación de mensajes o conocimiento" (Campos, 2006, p. 19). Se ajusta a la perspectiva de que el ser humano aprende ampliamente a través de las experiencias y representaciones visuales.

Campos expresa que "Bower demostró que los seres humanos somos capaces de realizar estas representaciones y cuando esto ocurre el aprendizaje es más eficaz" (Campos, 2006, p. 19). En su trabajo de investigación indicó que si una persona escucha o lee una historia y simultáneamente realiza una representación mental y luego gráfica, el aprendizaje y la retención serán mayores. Por la década de los 70 la única técnica gráfica que se utilizaba para organizar contenidos era el cuadro sinóptico.

Entonces Barrón introdujo el concepto de "organizador grafico" teniendo a la base a D. Ausubel, quien utilizaba un modelo de organizador pero era textual. Fueron Noverk y Ausubel quienes presentaron el "mapa Conceptual como una herramienta gráfica para posibilitar el aprendizaje significativo" (Campos, 2006, p. 19). "Tony 
Buzan inició trabajos en los años 60 sobre lo que se denomina mapas mentales... permiten gran libertad en su elaboración al hacer uso de conceptos y relaciones, líneas, iconos, colores, texto, etc." (Campos, 2006, p. 19). Entre los organizadores gráficos más conocidos, difundidos y usados se encuentran: el mapa mental, conceptual, sinóptico, diagrama de flujo, la línea del tiempo. De la Torre propone el hexagrama, además existen otros que potencian la enseñanza y aprendizaje de una manera didáctica y eficaz.

Se puede dar una relación estrecha entre la enseñanza y el aprendizaje, en donde "cada uno de los estudiantes se enfrenta con la situación de aprender. Aprender nuevos conocimientos, nuevas habilidades y muchas veces nuevas actitudes" (Amaya, 2010, p. 20). Por una parte se da un proceso de enseñanza y por otra el de aprender, que se dan en diversas situaciones en el salón de clase, del libro de texto, del campo de trabajo, de los mismos compañeros, docentes, etc., también se aprende en diferentes tiempos de la vida, para este caso se da un aprendizaje presencial y a la vez mediado por las TIC.

Para Hernández (2008), un "mapa conceptual es un instrumento mental; los instrumentos cognitivos son dispositivos, tanto mentales como informáticos, destinados al sostén, al estímulo, a la orientación, al análisis y a la extensión de las capacidades analíticas y de pensamiento de los estudiantes" (p. 52). Esta es justamente una herramienta visual que ayuda a facilitar la percepción, la guía y conlleva al análisis de un tema.

Se puede considerar como una actividad didáctica, una representación visual de la estructura cognitiva de un persona sobre un argumento. Es la comprensión de los contenidos como el resultado de una concepción de un tema y permite entender mejor una disciplina o materia, en donde un mapa conceptual es un soporte muy valioso para el estilo holístico del aprendizaje.

Entre las herramientas didácticas se encuentra el hexagrama, que es un "gráfico para efectos didácticos" (De la Torre, 2009, p. 155), el cual "cuenta con seis lados; en cada uno aparece un elemento distinto; así, en la parte superior central se encuentra el título o tema del hexagrama; en la parte superior izquierda, se localiza un mapa mental; en la parte superior derecha aparece el resumen de una lectura de comprensión; en la inferior izquierda una pregunta... " (De la Torre, 2009, p. 155), de tal manera que el hexagrama se constituye en un instrumento que permite adquirir conocimientos de un texto de forma didáctica.

Educación superior a distancia. La modalidad de educación a distancia surge como respuesta a las necesidades de jóvenes, adultos y profesionales que no pueden asistir a un sistema escolarizado. Los avances tecnológicos en materia de cómputo y 
telecomunicaciones ayudan a dar respuesta a dichas necesidades y se convierten en un apoyo para esta forma de educación, que se puede ofrecer en cualquier tiempo y en cualquier lugar, a través del uso de diversos medios de comunicación.

La educación a distancia es un reto y para que "funcione de manera óptima, se requiere una capacidad de autoaprendizaje en el alumno que, por lo general, en edades tempranas no posee, y debe adquirir durante la educación obligatoria" (De La Torre, 2009, p. 237). Es importante reconocer que esta modalidad o sistema se puede utilizar en los diferentes niveles educativos (preescolar, primaria, secundaria y superior en el nivel profesional), e impartir en instituciones públicas como privadas.

El sistema para la formación profesional, corresponde al "nivel educativo conocido como formación profesional, vocacional o técnica, tiene por finalidad específica la educación integral y su capacitación para el ejercicio de una profesión específica" (De La Torre, 2009, p. 239). Por lo tanto, la formación en un área técnica corresponde a la preparación o capacitación en áreas disciplinarias específicas, en pro de una consecución de un título de: auxiliar, técnico, tecnólogo o profesional, de todas maneras se especializa en algo definido.

\section{Diseño metodológico}

Esta propuesta se desarrolló en tres etapas: la primera se dio en el diseño de una encuesta para determinar qué tanto se conoce acerca de los mecanismos de protección de los derechos humanos.

La segunda, la participación en un foro virtual, con el fin de profundizar en el conocimiento y manejo de los mecanismos de protección de los derechos humanos en Colombia.

Una tercera etapa que se subdivide en tres momentos: el primero en torno a la fundamentación teórica de los derechos humanos y "mecanismos de protección", desde las diferentes perspectivas. En el segundo se diseñaron algunas estrategias de aprehensión de los siguientes mecanismos: derecho de petición, acción de tutela, habeas corpus, habeas data, acción popular, acción de grupo y acción de cumplimento y de las instituciones: Personería, Defensoría del pueblo y Procuraduría, de acuerdo con la Constitución de 1991; y el tercero corresponde al desarrollo de actividades de casos hipotéticos y reales.

Primera etapa: encuesta: esta fue aplicada a 66 estudiantes del Programa de Regencia de Farmacia. Corresponde a dos momentos: acerca de las generalidades de los encuestados y de diez preguntas tanto de selección como abiertas, que permiten conocer la voz de cada uno de ellos. 
Generalidades: respecto a generalidades se abordan aspectos como el sexo, la asignatura, el semestre y el Cread en donde se aplicaron las estudiantes, con el proposito de contextualizar la pequeña investigacion que se ha realizado y donde se ha aplicado la encuesta.

\begin{tabular}{|c|c|c|}
\hline \multicolumn{1}{|c|}{ Opción } & Cantidad & Porcentaje \\
\hline a Femenino & 59 & $89,4 \%$ \\
\hline b Masculino & 7 & $10,6 \%$ \\
\hline & 66 & $100,0 \%$ \\
\hline
\end{tabular}

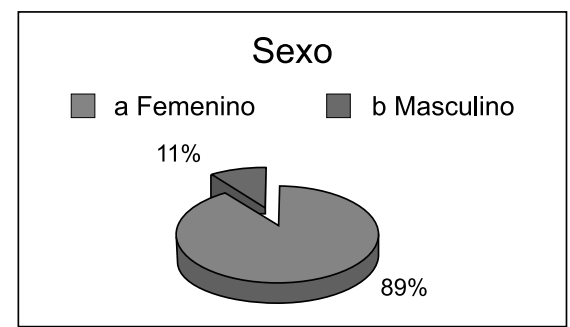

Se demuestra que el $89,4 \%$ corresponde a mujeres y el $10,6 \%$ a hombres. Se comprueba que cursan la tecnología en regencia de farmacia más personas del género femenino, y el personal en donde se aplicó la encuesta en un alto porcentaje es femenino.

Coincide la misma cantidad de estudiantes en la asignatura y el semestre, es decir, la misma cantidad de estudiantes de la asignatura de Ética, en el segundo semestre. Son 31 estudiantes de segundo semestre de Ética y 35 de la asignatura de Cultura Política de tercer semestre quienes ya van a la par, otra asignatura de Cultura Política y puede ser un aspecto importante para el estudio.

\begin{tabular}{|l|c|c|}
\hline \multicolumn{1}{|c|}{ Opción } & Cantidad & Porcentaje \\
\hline a. Duitama & 22 & $33,3 \%$ \\
\hline b.Sogamoso & 7 & $10,6 \%$ \\
\hline c. Tunja & 37 & $56,1 \%$ \\
\hline & 66 & $100,0 \%$ \\
\hline
\end{tabular}

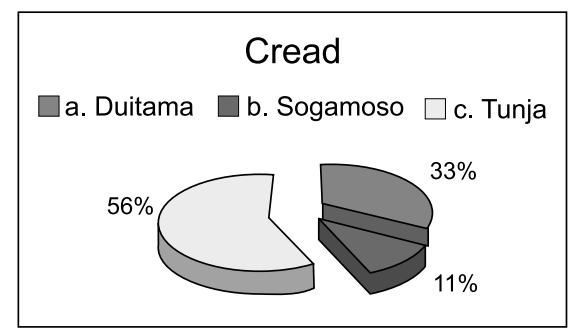

Respecto al Cread, el $56 \%$ de los estudiantes encuestados se encuentra en el Cread Tunja, esta ciudad es la capital de departamento por lo tanto puede incidir en el estudio. El $33 \%$ de la ciudad de Duitama y el $11 \%$ de Sogamoso, también se evidencia el número de estudiantes de cada Cread, en orden ascenderte o descendente que permite analizar el estudio

Son diez preguntas en las que se muestran en primer lugar aspectos generales de la Declaración Universal del Hombre y de los Derechos Humanos. Luego se ahonda en las instituciones que protegen los derechos humanos y en los mecanismos de protección de los mismos. 


\section{La Declaración Universal de los Derechos Humanos se dio en el año:}

\begin{tabular}{|l|c|c|}
\hline \multicolumn{1}{|c|}{ Opción } & Cantidad & Porcentaje \\
\hline a. 1945 & 10 & $15,2 \%$ \\
\hline b. 1948 & 17 & $25,8 \%$ \\
\hline c. 1958 & 23 & $34,8 \%$ \\
\hline d. 1789 & 10 & $15,2 \%$ \\
\hline No contesta & 6 & $9,1 \%$ \\
\hline & 66 & $100,0 \%$ \\
\hline
\end{tabular}

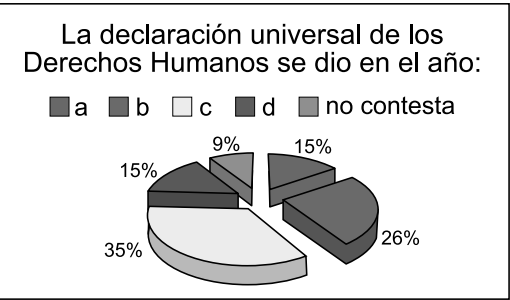

Se demuestra el desconocimiento por parte de los estudiantes de la fecha de la Declaración Universal de los Derechos Humanos, pese a que no se entró en detalle de más datos de la misma. 23 de los 66 estudiantes coinciden en que la fecha es en 1958 y realmente se desconocen las razones. Solo el $25 \%$ responde de manera afirmativa a lo que es en verdad la respuesta correcta. Es preocupante comprobar que el 75 \% desconoce el año de la Declaración de los Derechos Humanos.

\section{La Declaración Universal de los Derechos del Hombre se dio en el año:}

\begin{tabular}{|l|c|c|}
\hline \multicolumn{1}{|c|}{ Opción } & Cantidad & Porcentaje \\
\hline a. 1800 & 10 & $15,2 \%$ \\
\hline b. 1914 & 17 & $25,8 \%$ \\
\hline c. 1789 & 12 & $18,2 \%$ \\
\hline d. 1989 & 23 & $34,8 \%$ \\
\hline No contesta & 4 & $6,1 \%$ \\
\hline & 66 & $100,0 \%$ \\
\hline
\end{tabular}

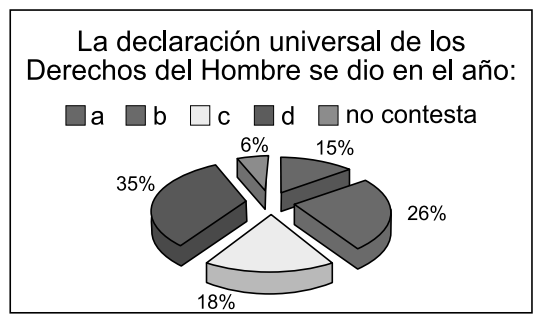

Se desconoce por parte de los estudiantes la fecha de la Declaración Universal de los Derechos del Hombre, solo 12 de los 66, es decir, el $18 \%$ de los estudiantes conoce la fecha correcta. Se evidencia que el $34,8 \%$ de los estudiantes contestaron la fecha de 1989, pero no hay argumentos sobre el desconocimiento de la fecha.

\section{Son rangos distintivos de los derechos humanos:}

\begin{tabular}{|c|c|c|c|}
\hline Opción & Cantidad & Porcentaje & \multirow{2}{*}{$\begin{array}{l}\text { Son rangos distintivos de los } \\
\text { Derechos Humanos }\end{array}$} \\
\hline a. Universales y particulares & 27 & $40,9 \%$ & \\
\hline b. Universales e incondicionados & 19 & $28,8 \%$ & \multirow{2}{*}{$\square \mathrm{a} \square \mathrm{b} \square \mathrm{c} \square \mathrm{d} \square$ no contesta } \\
\hline c. Subjetivos y relativos & 2 & $3,0 \%$ & \\
\hline d. Jurídicos y protegidos & 15 & $22,7 \%$ & \\
\hline No contesta & 3 & $4,5 \%$ & \\
\hline & 66 & $100,0 \%$ & \\
\hline
\end{tabular}


De los rasgos distintivos de los derechos humanos, los estudiantes consideran que los: "Universales y particulares" con el $40.9 \%$ de todas maneras es seguido por la respuesta correcta que es la b: "Universales e incondicionados".

4. Instituciones o instancias creadas para defender los derechos humanos: a. Defensoría del Pueblo, Procuraduría y Personería b. Habeas Corpus, Fiscalía y comisaria. c. Habeas Data, Gobernación y Presidencia de la Republicad. Juzgado, Acción de cumplimiento y Ministerio de Defensa.

\begin{tabular}{|l|c|c|}
\hline \multicolumn{1}{|c|}{ Opción } & Cantidad & Porcentaje \\
\hline $\mathrm{a}$ & 52 & $78,8 \%$ \\
\hline $\mathrm{b}$ & 5 & $7,6 \%$ \\
\hline $\mathrm{c}$ & 0 & $0,0 \%$ \\
\hline $\mathrm{d}$ & 7 & $10,6 \%$ \\
\hline No contesta & 2 & $3,0 \%$ \\
\hline & 66 & $100,0 \%$ \\
\hline
\end{tabular}

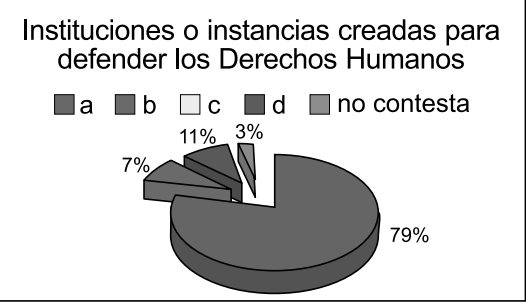

Frente a esta pregunta, los estudiantes en un 78,8 \% evidencian que las instituciones creadas en Colombia para defender los derechos humanos desde 1991, son "Defensoría del pueblo, Procuraduría y Personería".

5. ¿Qué son los derechos humanos? Como pregunta abierta, los estudiantes brindan la siguiente información, que se extrae de ella lo siguiente:

*Situaciones a las que se tiene derecho como la salud y la educación. *Son reglamentos impuestos para el cumplimiento del trato digno de las personas. ${ }^{*}$ Como la palabra lo dice es el derecho que tiene cada ser humano a la libre expresión, derecho al respeto a ser valorado como persona sin ser discriminados por nadie. ${ }^{*}$ Los derechos son igualdad, respeto de la integridad de cada ciudadano y el respeto de su pensamiento. *Son derechos que se adquirieron desde que nacemos y por obligación debe la sociedad hacer cumplir. *Las reglas que tiene todos para todos en cuanto a respeto *Son las libertades que tiene un ser humano para cumplir sus necesidades básicas para la dignificación. *Derecho a la vida son (sic) los cuales defienden a la persona ante atropellos de un determinado estado. *Son artículos que defienden a las personas contra cualquier arbitrariedad que atente contra su integridad. *Vida, educación, vivienda digna, salud, opinar libremente, libre creencia.

6. ¿Cómo educar en los derechos humanos? Como pregunta abierta, los estudiantes brindan la siguiente información, que se extrae de ella lo siguiente:

*A través de la casa y la educación en la escuela. *De una manera respetuosa y con valores para que de esta manera eduquemos seres educados para un mejor 
mañana. *Desde pequeños enseñar a nuestros hijos a respetar y valorar la vida. * Respetando a los demás y haciendo que los demás nos respeten. *Dando a conocer los derechos humanos. Educar de una forma respetuosa, amable y hacer que se cumplan los derechos.*Para educar cada uno de estos derechos debemos ser más tolerantes, ayudar al prójimo, para mejorar nuestra calidad de vida. ${ }^{*}$ Los podemos educar desde muy pequeños estos pueden venir de familia o se aprenden para el transcurso de la vida.*Por medio de concientización y obligando a los encargados a hacer cumplir. *Respetando los derechos de los niños especialmente. ${ }^{*}$ Los podemos educar desde casa viendo en la familia de que necesidades carece. ${ }^{*}$ Cumpliendo y dando un buen ejemplo a ellos para que los demás lo eduquen.*Velando por nuestros derechos y reclamando ante cualquier rechazo.

7. ¿Qué son los mecanismos de protección de los derechos humanos? Como pregunta abierta, los estudiantes brindan la siguiente información, que se extrae de ella lo siguiente:

*Son artículos que protegen los derechos como una acción de tutela o peticiones. *Los mecanismos son una ayuda para hacer cumplir los derechos del pueblo y su población en general.*Son el medio que defiende los derechos de la persona.*Son los que ayudan a las personas en caso de abusos e injusticias. ${ }^{*}$ En caso de cualquier problema no me pueden negar mis derechos. *Dando capacitación a las personas y a los niños y enseñarles cuales son los derechos. *Están constituidos por diferentes medios lo cual proporcionan una solución a inconvenientes en el entorno social.*Son las formas que tiene cada persona para poder protegerse de cualquier posible violación de derechos.

*Son operaciones creadas para proteger y defender aunque también obligar al cumplimiento de los derechos humanos estipulados en la constitución. *Mediante el defensor del pueblo son entidades dedicadas a la defender los DDHH. *Son un grupo de personas que estudian sobre la protección de los derechos humanos para así darnos a entender y aprender que como personas también tenemos una protección en cualquier caso del pueblo. Encargados de velar por el cumplimiento de los derechos sin que sean vulnerados*Son entes que nos ayudan para defender nuestros derechos cuando han sido vulnerados y violados *Son organización que se encargan por velar por cada uno de nuestros derechos. *Son aquellos encargados para hacer valer nuestros derechos.

8. ¿Cómo educar en los mecanismos de protección de los derechos humanos? Como pregunta abierta, los estudiantes brindan la siguiente información, que se extrae de ella lo siguiente:

* Para educar cada uno de estos derechos es necesario que conozcamos cada derecho como la vida o la libre expresión. *Haciendo respetar las normas y reglas que hay 
para las personas que quedan al frente de sus pueblos. *De igual manera es importante involucrar los mecanismos de protección desde la escuela.*Aplicando y defendiendo los mismos *Se puede educar dependiendo el grado de protección de la cada mecanismo ofrece ya que los derechos humanos son indispensables en la convivencia y sociedad.*A través de capacitaciones y así dar a entender la protección de los derechos humanos es muy importante para nosotros los seres humanos.*Mediante la práctica de estos. *Dando a conocer cada uno de los mecanismos y los derechos a través de diferentes medios y que sean respetados. *Dándoles a conocer al pueblo y actualizarse en los cambios (sic). ${ }^{*}$ La forma más fácil educar, desde la casa, el colegio hasta la universidad. *Mediante la formación educativa, esta debe ser desde cuando la persona empieza a vivir, es decir, desde que es niño.

\section{Escriba los mecanismos de protección de los derechos humanos que usted conozca:}

\begin{tabular}{|l|c|}
\hline \multicolumn{1}{|c|}{ Opción } & Cantidad \\
\hline a. A. Tutela & 40 \\
\hline b. D. Petición & 23 \\
\hline c. H. Corpus & 36 \\
\hline d. H. Data & 26 \\
\hline e. A. Cumpli & 14 \\
\hline f. A. Popular & 2 \\
\hline g. A. Grupo & 2 \\
\hline h. Otros & 61 \\
\hline i. No contesto & 5 \\
\hline j. Ninguno & 23 \\
\hline k. Todos & 0 \\
\hline Total & 66 \\
\hline
\end{tabular}

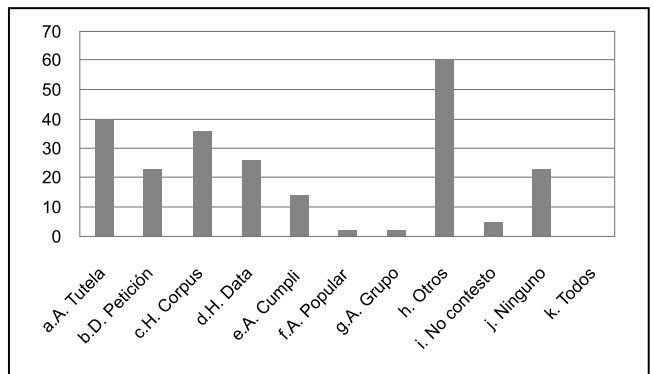

Para esta pregunta, que es tal vez muy elemental para el estudio pues se trata de abordar los mecanismos protección de los derechos humanos en Colombia. De entrada se evidencia que ninguno de los estudiantes conoce los siete mecanismos protección, que 23 de los 66 estudiantes no conoce ninguno. Que cuarenta estudiantes conocen invitados que la acción de tutela es un mecanismo protección, tal vez como el más conocido y quizás usado, seguido en conocimiento el habeas corpus y luego el habeas data, posteriormente el derecho de petición. Pero verdaderamente se comprueba que ellos no conocen la acción de grupo ni la acción popular. Esta situación es propicia para iniciar un proceso de formación desde la pedagogía, pues no se requiere que sea una asignatura: Constitución Política de Colombia, pues es natural del ciudadano conocer sus derechos y desde luego los mecanismos e instituciones que los defienden. 
10. Desde la pedagogía; ¿Cómo se pueden abordar los derechos humanos? Como pregunta abierta, los estudiantes brindan la siguiente información, que se extrae de ella lo siguiente:

*Los derechos se abordan a partir de cada uno de los valores que tengamos desde casa. *Tener claro los derechos y sus mecanismos para hacerlos cumplir.*Los podemos abordar haciendo (sic) charlas a las personas. *Dando conferencias o charlas para que así sepa la gente de estos. *Centrando más el tema en las clase ya que de eso sabemos poco. ${ }^{*}$ Conociendo de ante mano la definición, los mecanismos de control, funciones y demás definiciones que garantizan.*Primero leyendo detenidamente cuales son los derechos humanos y cómo podemos familiarizarnos con ellos y como se están discriminando algunos de ellos.*Desde las instituciones y las universidades capacitar y enseñar a las personas sobre los Derechos humanos y sus mecanismos de protección. *Por medio de juegos didácticos ya sean canciones, cuentos, dramatizaciones, etc.

Segunda etapa: foro virtual, con el fin de profundizar en el conocimiento y manejo de los mecanismos de protección de los derechos humanos en Colombia. Con la siguiente pregunta: ¿Cuál es la importancia de los derechos humanos y cuál es la realidad de los mismos en Colombia? y ¿Por qué son importantes los mecanismos de protección de los derechos humanos en Colombia y si se aplican?

La participación de los estudiantes en el foro fue activa, de compromiso y donde ya hay más conocimiento de la realidad de los derechos humanos en Colombia y su importancia. Los estudiantes reconocen la importancia de estos pero, a la vez, expresan la no aplicación de los mismos en nuestro país. Fue una oportunidad de investigar e ir madurando en el conocimiento y manejo de los mecanismos de protección.

\begin{tabular}{|l|c|c|}
\hline \multicolumn{1}{|c|}{ Opción } & Cantidad & Porcentaje \\
\hline a. Duitama & 22 & $33,3 \%$ \\
\hline b. Sogamoso & 7 & $10,6 \%$ \\
\hline c. Tunja & 37 & $56,1 \%$ \\
\hline & 66 & $100,0 \%$ \\
\hline
\end{tabular}

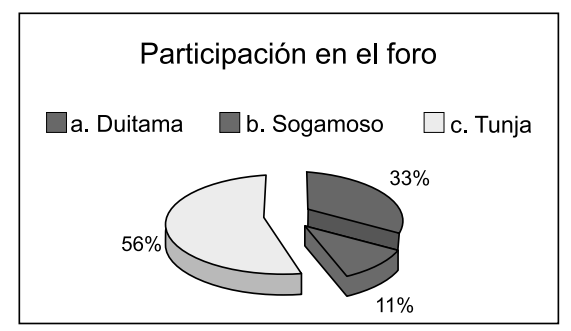

Se muestra también la plataforma bajo el esquema de la Universidad Pedagógica y Tecnológica de Colombia, la pregunta del foro y se alcanza a observar la participación de algunos de los estudiantes de los diferentes Cread. 


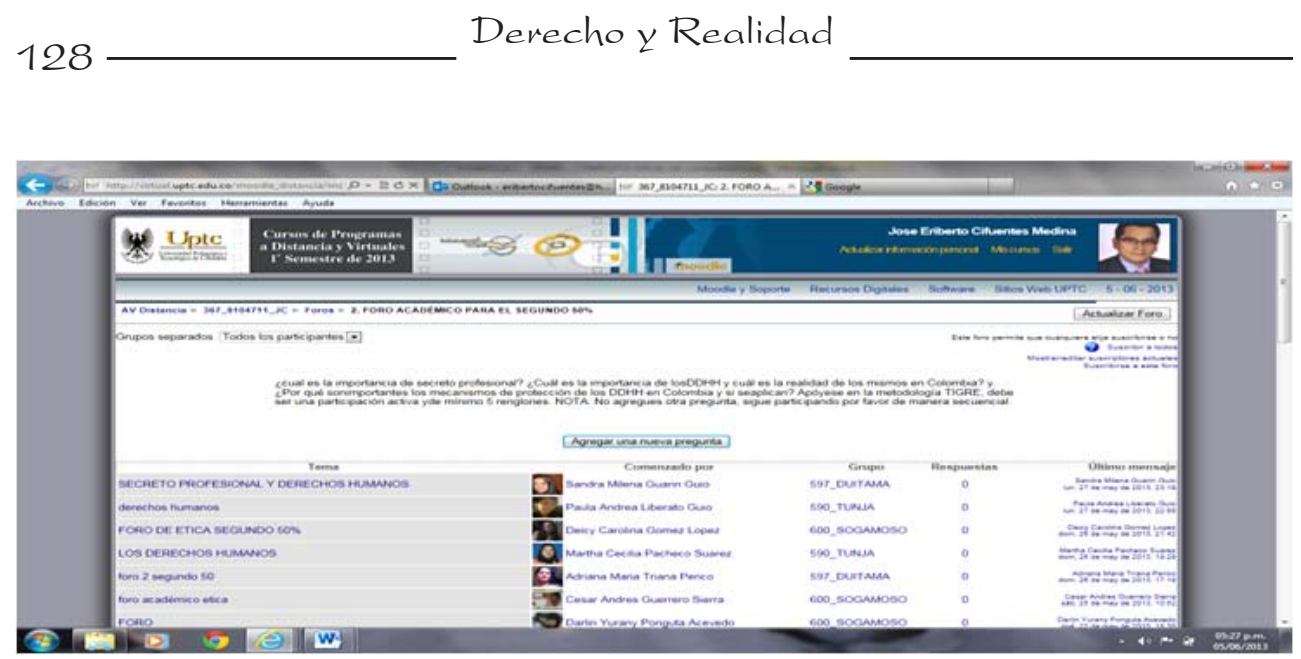

Fuente: http://virtual.uptc.edu.co/moodle_distancia/mod/forum/view.php?id=25315\&group $=0$

A continuación se muestran los comentarios de algunos estudiantes, uno por Cread, con el fin de conocer de primera mano los aportes de ellos y el progreso entre la primera y la segunda etapa, para consolidar una tercera.

¿Cuál es la importancia de los derechos humanos? "Los derechos humanos son muy importantes ya que su función principal es la de garantizar, velar y promover una sana convivencia entre las personas de un país sin tener ningún tipo de discriminación de sexo, raza, estrato, creencia etc. así mismo como un ciudadano recibe derechos de igual manera debe cumplir con unos deberes donde estos proporcionan una sana convivencia a las demás personas”.

¿Cuál es la realidad de los mismos en Colombia? "Usualmente se suele ver demasiadas injusticias tanto de parte de los ciudadanos como del propio gobierno, la constitución política de Colombia tiene escrito los determinados derechos que tenemos todos los ciudadanos pero cómo es posible observar a padres pagando la educación de sus hijos cuando existe el derecho a la educación o una simple vista es la de la salud, hay un indeterminado número de personas que mueren a diario por la negligencia por parte de las empresas encargadas de prestar atención a las personas cuando también existe el derecho a la salud. En tal es muy importante conocer los derechos humanos para que no se vean injusticias y que si se comenten sepamos como reclamar y exigir los derechos que tenemos y que brinda la constitución".

¿Por qué son de importancia los medios de protección de los derechos humanos en Colombia y si se aplican? "Existen medios encargados de proteger y velar los derechos humanos de los colombianos para garantizar una confortable y digna convivencia; entre los cuales están: Acción Popular, Derecho de Petición. Habeas, Corpus, Habeas Data, Acción de Cumplimiento, Acción de Grupo y Acción Popular”. (N. P. Cread Tunja) 
"Es una manera o prototipos de leyes para llevar una buena convivencia en un municipio, en el país, y a nivel mundial, nos sirve para escuchar y ser escuchados. Son los derechos que adquirimos cuando nacemos hasta que morimos en Colombia los derechos humanos en la mayoría de ocasiones son vulnerados ya que no todo el mundo tiene claro a que tiene derecho y cuáles son sus obligaciones. Son importantes porque todos estamos obligados a cumplir y respetar lo establecido en la constitución política que nos rige, para poder tener una sana convivencia dentro de ciertas normas y límites”. (M. E. Cread Chiquinquirá)

"La importancia de los derechos humanos es muy necesaria, gracias a ellos tenemos un respaldo frente a determinadas situaciones presentadas en nuestro diario vivir; con ayuda de las autoridades competentes. Estos mecanismos tienen como función proteger a las personas de los abusos de los estados, asignando a un grupo de organismos encargados de defender estos derechos”. (M. Z. Cread Duitama)

"Los derechos humanos son los que posee toda persona por el simple hecho de haber nacido y es el sustento de la dignidad humana, sin ningún tipo de discriminación. Para apreciar la importancia de estos debemos ponernos en lugar de aquellos que no los poseen. En Colombia desafortunadamente no tenemos la suficiente información de cuáles son nuestros derechos y solo acudimos a ellos cuando nos ocurre algún incidente no nos tomamos la tarea de empaparnos sobre el tema, los casos más frecuente son con respecto al sector de la salud es pésimo es decir las personas que necesitan algún medicamento de control o necesitan alguna cirugía son más los tramites que tiene que realizar para que le otorguen sus necesidades y a veces sin una respuesta positiva realmente para mi concepto la injusticia a un permanece y mucho más si tú no tienes los recursos necesarios para defenderte" (Z. S. Cread Sogamoso)

Tercera etapa: se subdivide en tres momentos, el primero en torno a la fundamentación teórica de los derechos humanos y "mecanismos de protección", desde las diferentes perspectivas; en el segundo se diseñaron algunas estrategias de aprehensión de los mecanismos y de las instituciones que de acuerdo con la Constitución de 1991, protegen los derechos humanos; y el tercero corresponde al desarrollo de actividades de casos hipotéticos y reales de la temática.

Primer momento: corresponde a una breve definición de cada uno de los mecanismos de protección de los derechos humanos.

1. La acción de tutela: la acción de tutela nace con la Constitución de 1991, en su artículo 86 "toda persona tendrá acción de tutela para reclamar ante los, por sí misma o por quien actúe en su nombre, la protección inmediata de sus derechos constitucionales fundamentales, cuando quiera que estos resulten vulnerados o 
amenazados por la acción o la omisión de cualquier autoridad pública”. Se contempla como un mecanismo, instrumento o herramienta orientada a la protección rápida e inmediata de los derechos fundamentales consagrados en la Constitución, del artículo 11 al 41.

2. El habeas corpus: se creó para contrarrestar las arbitrariedades y atropellos de la fuerza pública. Es el derecho que tiene la persona de pedir ante un juez que decida si en la detención ocurrieron irregularidades que provocan que se declare el procedimiento ilegal. Cuando cualquier autoridad ya sea judicial, policial o militar, hace una detención, debe cumplir una serie de requisitos que por ley.

3. El derecho de petición: el derecho de petición es un derecho fundamental que tiene todo ciudadano de hacer solicitudes respetuosas para conocer asuntos que pueden ser de su incumbencia a las autoridades públicas o particulares que presten servicios públicos; se encuentra consagrado dentro del capítulo de los derechos fundamentales de la Constitución Nacional, en el artículo 23.

4. La acción de cumplimiento: la acción de cumplimiento surge con la Constitución de 1991, la cual en su artículo 87 contempla "que toda persona podrá acudir ante autoridad judicial para hacer efectivo el cumplimiento de una ley o un acto administrativo". En caso de prosperar la acción, la sentencia ordenará a la autoridad renuente el cumplimiento del deber omitido. Esta acción de cumplimiento se da a conocer a partir de la ley 393 de 1997 que la reglamentó y estableció los límites y alcances de dicha ley.

5. La acción popular: en el artículo 88 de la Constitución Nacional se crean las acciones populares y se regulan según la ley 472 de 1998. El objetivo es garantizar la defensa y protección de los derechos e intereses colectivos, así como los de un número plural de personas

6. El habeas data: está contemplado en el artículo 15 de la Constitución Política, no es propiamente un mecanismo de defensa, es en sí un derecho que tiene todo ciudadano para conocer, actualizar y rectificar informaciones que sobre él se hayan recogido en banco de datos y en archivos de entidades públicas o privadas.

7. La acción de grupo: busca la indemnización de perjuicio (s) ocasionado(s) a por los menos 20 víctimas que se encuentran en condiciones uniformes respecto a la causa que generó el daño.

Segundo momento: se diseñaron algunas estrategias de aprehensión de los siguientes mecanismos, tal vez la más usada fue la del mapa conceptual que permite apropiar de manera esquemática elementos conceptuales. 1. La acción de tutela, 2. El habeas 
corpus, 3. El derecho de petición, 4. La acción de cumplimiento, 5. La acción popular, 6. El habeas data y 7. La acción de grupo:

Veamos algunos ejemplos de estrategias pedagógicas, didácticas y metodológicas, con el propósito de visualizar de una forma más clara cada el mecanismo de protección de los derechos humanos en Colombia.

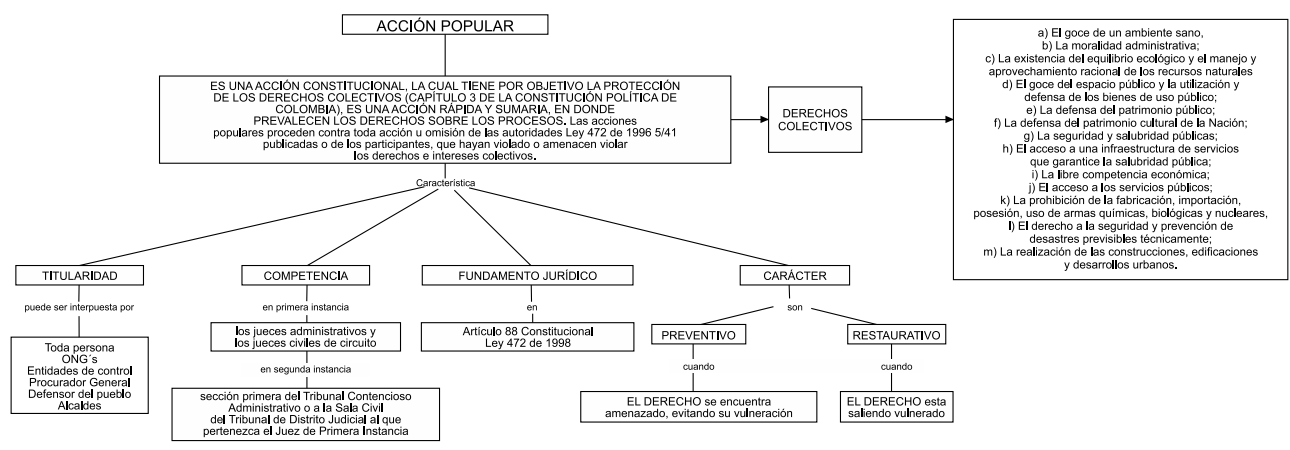

Fuente: mapa conceptual elaborado por Paula - Cread Tunja

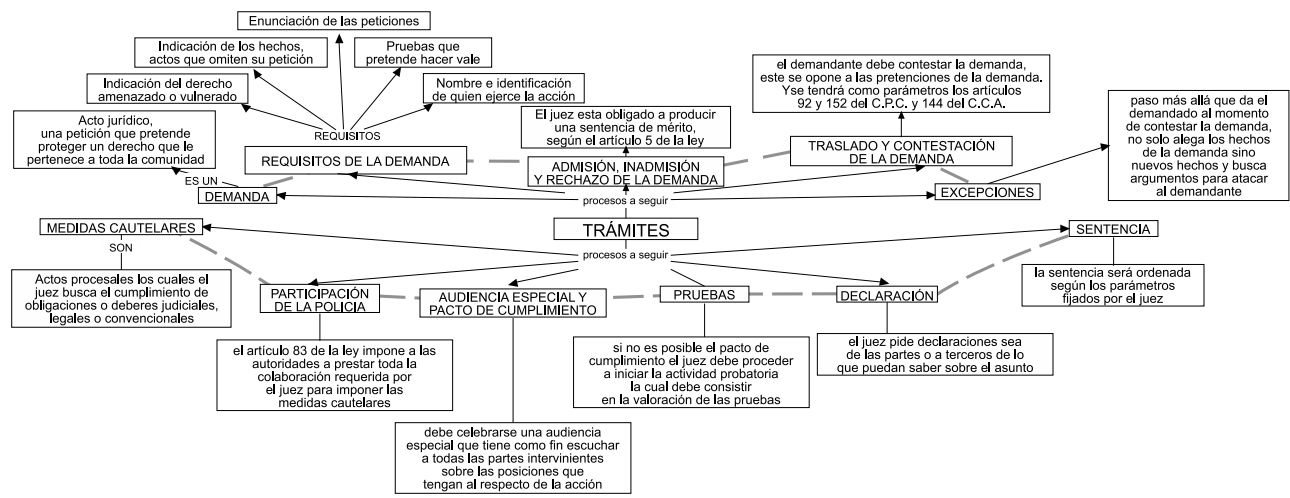

Fuente: mapa conceptual elaborado por Paula - Cread Tunja

Tercer momento: corresponde al desarrollo de actividades de casos hipotéticos y reales en los que se trató la temática. Veamos un ejemplo:

\section{El derecho de petición:}

Doctor (a): (A quien se dirige)

E.S.D Referencia: DERECHO DE PETICIÓN 
Nosotros, como integrantes de (Nombre de la organización a la que pertenecemos o nombre e identificación de quien hace la acción) hacemos uso del DERECHO DE PETICIÓN consagrado en el artículo 23 de la Constitución Política de Colombia y en el artículo 9 y siguiente del Código Contencioso Administrativo con el fin de solicitarle:

(Lo que se solicita, recordemos que podemos pedir información, que se haga o deje de hacer). Cualquier comunicación favor dirigirla (Dirección donde pueden darnos respuesta)

Atentamente, Firma de quien o quienes hacen la petición

\section{Conclusiones}

Los resultados han sido valiosos para el ejercicio de la labor docente respecto a la fundamentación de los derechos humanos en su historia, manejo instructivo de los mecanismos al igual que de la Constitución de 1991. En cuanto al proceso formativo de los estudiantes, se vislumbraron logros en redacción, apropiación, manejo de las formas jurídicas. A partir de esta propuesta ellos son capaces de afrontar la defensa de los derechos humanos, la fundamentación teórica, ya que están abiertos al conocimiento y la reflexión.

El diseño y aplicación de la encuesta fue tal vez la forma más eficiente de llevar a cabo un diagnóstico, donde se pudieron demostrar las diferentes falencias acerca de los derechos humanos y de sus mecanismos de protección, lo que se convirtió en el punto de partida. La posterior aplicación del foro virtual en la plataforma virtual, evidencia que ha mejorado el conocimiento de los mecanismos de protección de los derechos humanos.

La fundamentación teórica de cada mecanismo, de mano con los derechos humanos y de la Constitución, ha permitido elaborar estrategias pedagógicas y didácticas para afinar su manejo. También las guías para presentar cada uno y su posterior ejercicio práctico, permiten combinar la teoría y la práctica. De ahí el éxito desde la pedagogía, entre la enseñanza y el aprendizaje, el diálogo entre el aula de clase y la realidad, del docente que sin ser abogado conlleva la formación ciudadana en el conocimiento de la protección de los derechos humanos. 


\section{Referencias}

Amaya, G. J. et al. (2010). Estrategias de aprendizaje para universitarios. Un enfoque constructivista. ( $1^{\mathrm{a}}$ ed.). México: Trillas.

Campos, A. (2006). Organizadores gráficos. Técnicas visuales para aprender y enseñar. Revista Internacional Magisterio. Educación y pedagogía, 18, 2730 .

Cifuentes, M. E. (2001). ¿Qué son los derechos humanos? (1 ${ }^{\mathrm{a}}$ ed.). Bogotá: Red de Promotores de los Derechos Humanos, Defensoría del Pueblo, Editora Nacional.

Conde, S. (2004). La educación ciudadana. Revista Internacional Magisterio. Educación y pedagogía, 9, 28-30.

Constitución Política de Colombia (1991). Edición actualizada año 2008. Bogotá: Momo.

De la Torre, F. (2009). 12 Lecciones de pedagogía, educación y didáctica. $\left(4^{\mathrm{a}}\right.$ ed.). México. Alfa omega.

Hernández, F. V. (2008). Mapas conceptuales. La gestión del conocimiento en la didáctica. ( $2^{\mathrm{a}}$ ed.). México: Alfa omega.

Morales, G. (2005). Cuatro tesis para educar en derechos humanos. Revista Internacional Magisterio. Educación y pedagogía, 13, 27-30.

Tamayo, J. J. (2005). 10 palabras clave sobre derechos humanos. ( $1^{\mathrm{a}} \mathrm{ed}$.). Navarra España: Verbo Divino. 